Carnets de géographes

GÉOGRAPHES

\title{
Bien gérer les « espaces de natures », une éthique du faire-avec
}

Propositions pour une géographie des associations hétérogènes

Claire Tollis

\section{OpenEdition}

\section{Journals}

Édition électronique

URL : http://journals.openedition.org/cdg/950

DOI : $10.4000 /$ cdg.950

ISSN : 2107-7266

\section{Éditeur}

UMR 245 - CESSMA

\section{Référence électronique}

Claire Tollis, "Bien gérer les « espaces de natures », une éthique du faire-avec », Carnets de géographes [En ligne], 6 | 2013, mis en ligne le 01 septembre 2013, consulté le 24 septembre 2020. URL : http:// journals.openedition.org/cdg/950 ; DOI : https://doi.org/10.4000/cdg.950

Ce document a été généré automatiquement le 24 septembre 2020.

\section{c) (i) $९$}

La revue Carnets de géographes est mise à disposition selon les termes de la Licence Creative Commons Attribution - Pas d'Utilisation Commerciale - Pas de Modification 4.0 International. 


\section{Bien gérer les « espaces de natures ", une éthique du faire-avec}

Propositions pour une géographie des associations hétérogènes

\section{Claire Tollis}

1 La « crise environnementale » médiatisée sous la forme d'une série de dommages et de menaces qui pèsent sur la survie de notre espèce (Larrère, 1997) est à la fois le symptôme et le moteur de changements profonds : elle oblige nos sociétés à prendre en compte des entités et des phénomènes qui ne sont pas humains et que nous avions exclus de notre sphère morale. Plus largement, ces enjeux questionnent la façon dont nous nous organisons pour agir dans l'espace que nous partageons de facto avec une pluralité d'entités. Cette recherche doctorale s'intéresse plus particulièrement aux pratiques de gestion des "espaces de nature », ces espaces publics que l'on concède ou consacre à la "nature ». Les gestionnaires de ces espaces sont amenés à répondre à une multiplicité de demandes et d'injonctions pour "écologiser» leurs façons de faire. Cette mutation est délicate parce qu'elle place les gestionnaires dans une position où ils doivent faire des choix. Ils sont amenés à se questionner sur la légitimité, la faisabilité et l'acceptabilité de nouvelles mesures.

2 Nous observons et décrivons la mise en place de quatre initiatives se disant «éthiques » ou «responsables", c'est à dire des initiatives que nous qualifions d'éthiquetées: la suppression progressive de l'usage des produits chimiques dans les espaces verts de Grenoble, la construction d'un collectif de nettoyage des décharges dites "sauvages » dans le massif de la Chartreuse, l'aménagement d'une entrée du Parc National des Pyrénées et l'instauration de quotas de visiteurs dans la Mt Jefferson Wilderness (Oregon).

3 Pour suivre les acteurs au plus près de leurs activités quotidiennes, nous avons mis à profit plusieurs techniques d'enquête : une centaine d'entretiens a été réalisée avec les gestionnaires (responsables, techniciens, jardiniers, gardes), ainsi que plus de 200 questionnaires auprès des visiteurs et une analyse fine de plusieurs types de documents (comptes rendus de réunion, lettres de plainte, cartes, presse professionnelle, articles de journaux grand public). Nous avons également passé une trentaine de journées 
d'observation directe ou participante auprès des gestionnaires, que ce soit dans leurs bureaux, en réunion ou même sur le terrain.

4 Il apparaît que la responsabilité du devenir des "espaces de nature » n'est pas uniquement celle des gestionnaires : elle est distribuée le long d'une chaîne d'humains et de non-humains. Les acteurs comptent sur une pluralité de personnes, d'êtres et de choses pour maintenir ces espaces. Leurs pratiques relèvent d'expérimentations tout à la fois scientifiques, politiques et morales (Latour, 1995, Hache, 2011). Ils construisent des compromis situés et précaires, selon une éthique du faire-avec.

Dans ce mouvement, les parcs et jardins - comme figures de la séparation et de la domestication - cessent d'être l'échelle pertinente de réflexion et d'action: d'autres formes socio-spatiales émergent. Une géographie des associations hétérogènes (Murdoch, 1997) nous apparaît alors la plus à même de rendre compte des liens, des emboîtements d'échelles et des évènements éthiques fragmentaires qui se jouent dans le travail continu de ces collectifs s'adonnant à construire un monde commun constamment renégocié.

6 L'analyse des liens entre humains et non humains constitue un objet de recherche fécond pour explorer les questionnements propres à la géographie et plus largement aux sciences sociales. Ce sont, le plus souvent, des objets qui connectent les acteurs entre eux et leur permettent de passer à l'action, d'opérer des changements et d'acter ces derniers.

7 Cette approche permet de recoudre entre elles les échelles (micro/macro, individus/ société, structure/interactions) trop souvent traitées séparément mais aussi les sciences sociales et les sciences dures avec l'intégration d'êtres dont le fonctionnement ne peut être compris sans les apports de biologistes, par exemple. La thèse s'inscrit, en cela, dans une dynamique intégrative plus large, portée en particulier par la jeune recherche (Mounet, 2008 ; Créton-Cazanave, 2011 ; Obin, 2013).

8 L'originalité de cette thèse tient à l'exploration d'un objet, l'éthique dans sa forme actée, jusqu'ici peu étudié par la géographie. Se faisant, elle fait appel à un important corpus théorique issu de la philosophie, de l'anthropologie politique, des théories de l'action, mais aussi de la psychologie sociale et de la sociologie pragmatiste, aussi bien en français qu'en anglais. Elle apporte ainsi un éclairage important sur cet objet à travers la figure du compromis, principalement.

point de vue théorique et même épistémologique, ce travail participe également à

reformuler la question de ce qu'est la « nature » et ce que deviennent les espaces dans lesquels on tente de la parquer.

10 Enfin, la thèse propose une approche originale des processus participatifs émergents, qui sont concomitants de la crise environnementale dans laquelle de nouveaux publics s'invitent mais aussi sont invités pour discuter des issues souhaitables et les mettre en œuvre (bénévolat).

11 Nous ne cherchons pas à juger de la moralité des initiatives que nous suivons. L'intérêt que nous leur portons tient à l'évaluation que les acteurs pris dans ces initiatives font : ils les évaluent comme étant « responsables». Pour eux, il s'agit d'une bonne façon de gérer les espaces dont ils ont la charge. Nous cherchons à savoir comment ils arrivent à formuler ce jugement et ce qu'ils prennent en compte pour le faire. Nous mettons au jour certaines façons qu'ils ont d'arbitrer entre des intérêts pluriels et les «dettes morales » qu'ils contractent à l'endroit de ceux qu'ils n'ont pas 
encore pu satisfaire, par une description scrupuleuse des processus d'inclusion/ exclusion/reconnaissance qu'ils pilotent. Dans cette approche résolument descriptive qui renoue en quelque sorte avec l'ancienne géographie - il ne s'agit pas d'expliquer trop rapidement mais bien plutôt de donner à voir les attachements des acteurs et la façon dont ils font sens de leurs monde.

\author{
Lien électronique de la thèse en ligne \\ http://tel.archives-ouvertes.fr/tel-00814246

\section{Discipline} \\ Géographie
}

\title{
Directeurs de thèse
}

Philippe Bourdeau et Romain Lajarge, Université de Grenoble, Laboratoire PACTETerritoire

\section{Université}

Université de Grenoble

\section{Membres du Jury de thèse, soutenue le 10 décembre 2012}

Cynthia Ghorra-Gobin (Rapporteur et Présidente), Directrice de recherche au CNRS, IHEAL, Paris.

James D. Proctor (Rapporteur), Professor of Environmental Studies, Lewis \& Clark College, Portland.

Jean-Jacques Delannoy (examinateur), Professeur des Universités, Université de Savoie, Chambéry.

Jacques Lévy (examinateur), Professeur ordinaire de Géographie, EPFL, Lausanne. Valérie November, Directrice de recherche au CNRS, LATTS, Marne La Vallée.

Situation professionnelle à l'issue de la thèse

Chercheuse associée au laboratoire PACTE, Grenoble

Courriel de l'auteur

clairetollis@gmail.com

\section{BIBLIOGRAPHIE}

Créton-Cazanave L. (2010) Penser l'alerte par les distances. Entre planification et émancipation, l'exemple du processus d'alerte aux crues rapides sur le bassin versant du Vidourle, Thèse de doctorat, Université J. Fourrier, Grenoble, 384 p.

Hache E. (2011), Ce à quoi nous tenons, propositions pour une écologie pragmatiste, La Découverte, Paris, 246 p. 
Larrère C. et Larrère R. (1997) Du bon usage de la nature, pour une philosophie de l'environnement, Alto Aubier, Paris, $312 \mathrm{p}$.

Latour B. (1995) « Moderniser ou Ecologiser ? A la recherche de la septième cité », Ecologie politique, $\mathrm{n}^{\circ} 13$, pp 5-27.

Mounet C. (2007) Les territoires de l'imprévisible, Conflits, controverses et "vivre ensemble" autour de la gestion de la faune sauvage. Le cas du loup et du sanglier dans les Alpes françaises, thèse de doctorat en Géographie, Institut de Géographie Alpine, Grenoble, 564 p.

Murdoch J. (1997) « Towards a geography of heterogeneous associations », Progress in Human Geography, no 21, 3, p. 321-337.

Obin O. (2013) Territoires en construction. De la géographie sociale à l'acteur-réseau. Une lecture des dynamiques sportives de nature dans les Grands Causses, Thèse de doctorat en Géographie de l'Université de Grenoble

INDEX

Thèmes : Carnets de soutenances 